\title{
Nuclear Induction
}

\author{
F. BLOCH \\ Stanford University, California \\ (Received July 19, 1946)
}

\begin{abstract}
The magnetic moments of nuclei in normal matter will result in a nuclear paramagnetic polarization upon establishment of equilibrium in a constant magnetic field. It is shown that a radiofrequency field at right angles to the constant field causes a forced precession of the total polarization around the constant field with decreasing latitude as the Larmor frequency approaches adiabatically the frequency of the $r-f$ field. Thus there results a component of the nuclear polarization at right angles to both the constant and the $r-f$ field and it is shown that under normal laboratory conditions this component can induce observable voltages. In Section 3 we discuss this nuclear induction, considering the effect of external fields only, while in Section 4 those modifications are described which originate from internal fields and finite relaxation times.
\end{abstract}

\section{INTRODUCTION}

$T$ HE method of magnetic resonance ${ }^{\mathrm{l}}$ has been successfully applied to measure the magnetic moment of the neutron ${ }^{2}$ and of various nuclei. ${ }^{3}$ The principal feature of this method is the observation of transitions, caused by resonance of an applied radiofrequency field with the Larmor precession of the moments around a constant magnetic field. In its application to nuclear moments the deflection of molecular beams in an inhomogeneous field was used as a means of detecting the occurrence of nuclear transitions. This method of detection has proven to be very fruitful but it was clear, at the same time, that the connection between molecular beams and magnetic resonance was not of basic character. The question arose, in particular, whether nuclear transitions could not be detected by far simpler electromagnetic methods, applied to matter of ordinary density. ${ }^{4}$

An attempt in this direction was undertaken by Gorter and Broer ${ }^{5}$ whose arrangement was designed to indicate magnetic resonance absorption by a slight change in frequency of an electric oscillator. The experiment was based upon considerations which apply strictly to radiofrequency fields which are so small that

${ }^{1}$ I. I. Rabi, Phys. Rev. 51, 652 (1937).

${ }^{2}$ L. W. Alvarez and F. Bloch, Phys. Rev. 57, 111 (1940).

${ }^{3}$ I. I. Rabi, S. Millman, P. Kusch, and J. R. Zacharias, Phys. Rev. 53, 318 (1938); 55, 526 (1939).

${ }_{4}^{4}$ The first purely magnetic experiment to find an effect due to nuclear moments by measuring the susceptibility of liquid hydrogen was published by B. G. Lasarew and L. W. Schubnikow, Sov. Phys. 11, 445 (1937).

${ }_{5}$ C. J. Gorter and L. J. F. Broer, Physica 9, 591 (1942). they cause only a slight disturbance of the spin orientation; it was carried out with $\mathrm{LiCl}$ and $\mathrm{KF}$ at low temperatures and it was suggested that the failure to find an effect was caused by the fact that the nuclei had not found the orientation, corresponding to thermal equilibrium.

The first successful experiments to detect magnetic resonance by electromagnetic effects have been carried out recently and independently in the physics laboratories of Harvard ${ }^{6}$ and Stanford ${ }^{7}$ Universities. The experiment of Purcell and his collaborators is very closely connected to that of Gorter and Broer, the main difference being that resonance absorption manifests itself in the change of $Q$ of an electric oscillator rather than in a change of frequency, and it presupposes, likewise, the necessity of only slightly perturbing $r-f$ fields.

The considerations upon which our work was based have several features in common with the two experiments, previously mentioned, but differ rather essentially in others. In the first place, the radiofrequency field is deliberately chosen large enough so as to cause at resonance a considerable change of orientation of the nuclear moments. In the second place, this change is not observed by its relatively small reaction upon the driving circuit, but by directly observing the induced electromotive force in a coil, due to the precession of the nuclear moments around the constant field and in a direction

${ }^{6}$ E. U. Purcell, H. C. Torrey, and R. V. Pound, Phys. Rev. 69, 37 (1946).

${ }^{7}$ F. Bloch, W. W. Hansen, and Martin Packard, Phys. Rev. 69, 127 (1946). 
perpendicular both to this field and the applied $\mathrm{r}-\mathrm{f}$ field. This appearance of a magnetic induction at right angles to the $r-f$ field is an effect which is of specifically nuclear origin and it is the main characteristic feature of our experiment. In essence, it is similar to the Faraday effect of rotation of the plane of polarization of light around a magnetic field, with the $r-f$ field taking the place of the field vectors in a light wave and the observed perpendicular nuclear induction indicating a rotation of the total oscillating field around the constant magnetic field.

This effect is, of course, most outspoken at resonance (just as the Faraday effect becomes greatest in the neighborhood of a resonant frequency) and, in practice, is noticed by its sudden strong appearance under resonance conditions. It is worth while, however, to point out that the observation of nuclear induction should be possible even without any use of the magnetic resonance. Not only a weak $r-f$ field, acting at resonance over very many Larmor periods, can produce an appreciable nuclear change of orientation, but also a strong field pulse, acting over only a few periods. Once the nuclear moments have been turned into an angle with the constant field, they will continue to precess around it and likewise cause a nuclear induction to occur at an instant when the driving pulse has already disappeared. It seems perfectly feasible to receive thus an induced nuclear signal of radiofrequency well above the thermal noise of a narrow band receiver. It is true that, due to the broadening of the Larmor frequency by internuclear fields or other causes, this signal can last only a comparatively short time, but for normal fields it will still contain many Larmor periods, i.e., it will be essentially monochromatic. The main difference between this proposed experiment and the one which we have actually carried out lies in the fact that it would observe by induction the free nuclear precession while we have studied the forced precession impressed upon the nuclei by the applied $r-f$ field.

\section{NUCLEAR PARAMAGNETISM}

The existence of a resultant macroscopic moment of the nuclei within the sample under investigation is a common prerequisite for all electromagnetic experiments with nuclear mo- ments. It is in fact a change of orientation of this macroscopic moment which causes the observed effects, and irrespective of the changes of orientation of the individual nuclei which might be induced by a r-f field, their moments would always cancel each other, if they did so initially, and thus escape observation. In the experiments with molecular beams of Stern and Rabi this necessity is avoided by separation in the beam of nuclei with different orientation.

Even in the absence of any orientation by an external magnetic field one can expect in a sample with $N$ nuclei of magnetic moment $\mu$ to find a resultant moment of the order $(N)^{\frac{1}{2}} \mu$ because of statistically incomplete cancellation. This moment, however, would naturally be very small and in samples of normal size will be greatly increased as soon as the nuclei have found their equilibrium distribution in a field of a few thousand Gauss which, at the same time, will bring their Larmor precession into the convenient radiofrequency range.

In contrast to the familiar atomic paramagnetism which establishes itself almost immediately upon application of the polarizing field, there is no assurance for the same thing being true in the nuclear case. The time of establishment or "relaxation time" can be expected to vary anywhere between fractions of a second and many hours, depending in the most delicate manner upon the nuclear moments, the electronic structure of the atoms in the sample, their distance, and their motion. To study experimentally and theoretically this interesting relationship between nuclear relaxation time and atomic features seems to us, in fact, to be one of the fruitful fields of investigation which have now opened. ${ }^{8}$

It must be pointed out here that, in cases where the natural relaxation time should turn out inconveniently long, the establishment of thermal equilibrium can often be greatly accelerated by use of paramagnetic catalysts. The problem is similar to that of the conversion of

${ }^{8} \mathrm{~A}$ good deal of the theory of the paramagnetic relaxation time by I. Waller, Zeits. f. Physik 79, 370 (1932), R. deL. Kronig, Physica 6, 33, 1939. J. H. van Vleck, Phys. Rev. 57, 426 (1940), can readily be adopted to hold also for nuclear paramagnetism. For nuclear paramagnetism and its bearing on reaching low temperatures see E. Teller and W. Heitler, Proc. Roy. Soc. 155, 629 (1936). 
ortho- and parahydrogen which was found, for example to be accelerated by paramagnetic ions in solutions and by admixture of oxygen in the gas phase.

As to the nuclear induction effect, there exists the interesting possibility of first establishing the equilibrium in a strong magnetic field under conditions of relatively short relaxation time (by evaporation into an oxygen atmosphere, addition of a paramagnetic catalyst, heating, etc.), thereupon considerably lengthening the relaxation time (by recondensation, removal of the catalyst, cooling, etc.), and thus preserving the high field polarization for a considerable time, even when the sample is removed from the high field. A subsequent nuclear induction experiment, carried out under suitable conditions, can then exhibit either a moment, pertaining to the field in which it was originally established, or indicate that the relaxation time was comparable or short, compared to the time which has elapsed since removal. We shall come back later to some more considerations of the important role of the relaxation time, which are directly connected with our experiments.

For the following purposes we shall assume that the thermal equilibrium between the nuclear moments and their surrounding atoms has been actually established in an external magnetic field of strength $H$. Let $T$ be the equilibrium temperature and $n$ the number of nuclei per unit volume, each having a magnetic moment $\mu$ and an angular momentum $j h / 2 \pi$. We shall write

$$
M=\chi H,
$$

where $M$ is the resultant nuclear moment per cc in the field direction and where $\chi$ is the nuclear paramagnetic susceptibility. It is given by the familiar Curie formula

$$
\chi=[(j+1) / 3 j]\left(n \mu^{2} / k T\right),
$$

in which it is assumed that $H \mu \ll k T$. This condition will always be very well satisfied, except for extraordinarily strong fields or exceedingly low temperatures.

For a numerical example we shall take the protons in water at room temperature $\left(T=291^{\circ}\right)$. We have here $j=\frac{1}{2}$, i.e., $(j+1) / 3 j=1$, and with $\mu=1.4 \times 10^{-23}$ c.g.s., $n=6.9 \times 10^{22} \mathrm{~cm}^{-3}$, we obtain from (2) for the nuclear susceptibility

$$
\chi=3.4 \times 10^{-10}
$$

and with a field $H=10,000 G$ from (1)

$$
M=3.4 \times 10^{-6} \text { gauss }
$$

for the nuclear polarization.

While this value is small and would be difficult to observe directly, it is actually not $M$ but the rate of change of the rapidly varying nuclear induction $B=4 \pi M$, which is observed in our experiment and which we will show to be easily detectable.

\section{PRINCIPLE OF THE NUCLEAR INDUCTION}

We shall investigate the behavior of the great number of nuclei contained in a macroscopic sample of matter and acted upon by two external fields: a strong constant field and at right angles to it, a comparatively weak radiofrequency field. In order to simplify the explanation of the principle we shall, for the moment, omit some of the actually present complicating factors, and we shall assume:

(1) That the changes of orientation of each nucleus are solely due to the presence of the external fields;

(2) That the external fields are uniform throughout the sample.

The second assumption will evidently be justified with sufficient perfection of the experimental arrangement. It is the first assumption which is more serious; normally it will be far from being justified, and rather essential corrections will have to be introduced later to account for the actual conditions. The following conditions should be satisfied for its acceptance:

(1a) The atomic electrons do not cause appreciable fields to act upon the nuclei.

(1b) The interaction between neighboring nuclei can be neglected.

(1c) The thermal agitation does not essentially affect the nuclei, i.e., the relaxation time is long compared to the considered time intervals.

We shall come back to these three points in Section 4 ; accepting in this section the above assumptions, the discussion becomes comparatively simple: Let the $z$-direction be that of the constant field with strength $H_{0}$ and the $x$-direction that of the $r-f$ field with circular frequency $\omega$ 
and amplitude $2 H_{1}$, so that the total external field vector $\mathbf{H}$ has the components

$$
H_{x}=2 H_{1} \cos \omega t ; \quad H_{y}=0 ; \quad H_{z}=H_{0} .
$$

We shall further denote by $\mathbf{M}$ the vector, representing the nuclear polarization, i.e., the resultant nuclear moment per unit volume; it is the variation with time of this vector in which we are primarily interested.

To obtain this variation does not require the solution of the Schroedinger equation. It is enough to remember the general fact that the quantum-mechanical expectation value of any quantity follows in its time dependence exactly the classical equations of motion and that the magnetic and angular momenta of each nucleus are parallel to each other.

The resultant angular momentum vector $\mathbf{A}$ of all the nuclei, contained in a unit volume will, therefore, satisfy the classical equation

$$
d \mathbf{A} / d t=\mathbf{T},
$$

where $\mathbf{T}$ is the total torque, acting upon the nuclei and it is

$$
\mathbf{T}=[\mathbf{M} \times \mathbf{H}],
$$

where the vector $\mathbf{M}$ represents the resultant nuclear magnetic moment per unit volume. The parallelity between the magnetic moment $\boldsymbol{u}$ and the angular momentum a for each nucleus implies

$$
\boldsymbol{u}=\gamma \mathbf{a}
$$

with the gyromagnetic ratio

$$
\gamma=\mu / a
$$

and we have, therefore, also for the resultant quantities $\mathbf{M}$ and $\mathbf{A}$

$$
\mathbf{M}=\gamma \mathbf{A},
$$

with $\gamma$ likewise given by (9). ${ }^{9}$

Combining Eqs. (6), (7), and (10), we have, therefore, for the variation of the polarization vector $\mathbf{M}$

$$
d \mathbf{M} / d t=\gamma[\mathbf{M} \times \mathbf{H}] .
$$

For our purposes we are interested in a special solution of this equation which can be obtained

${ }^{9}$ Our treatment includes evidently both cases of $\boldsymbol{u}$ and a being parallel (i.e., having the same relative orientation as for a positive rotating charge) and opposite. Both are taken into account by assigning to the quantity $\gamma$ of Eq. (9) a positive or negative value respectively. We shall see below that the actual sign of $\gamma$ reveals itself in the phase of the induced voltage signal. if, for the fields $H_{0}$ and $H_{1}$ of Eq. (5) we have $H_{1} \ll H_{0}$, and if both are positive and constant. We shall further assume that the circular frequency $\omega$ of the $r-f$ field is in the neighborhood of the resonance frequency $\omega_{0}$, given by

$$
\text { i.e., that we have } \begin{gathered}
\omega_{0}=\gamma H_{0} \\
\left|\omega-\omega_{0}\right| \ll \omega_{0} .
\end{gathered}
$$

The actual oscillating field in the $x$-direction can then be effectively replaced ${ }^{10}$ by a field

$$
H_{x}=H_{1} \cos \omega t ; \quad H_{y}=\mp H_{1} \sin \omega t
$$

rotating around the $z$-direction with the sign of $H_{y}$ and, therefore, the sense of rotation being negative or positive, depending upon whether the sign of $\gamma$ is positive or negative.

It follows immediately from (11) that the magnitude $M$ of the polarization is constant. Besides, there is a special solution for which its $z$-component $M_{z}$ is likewise constant. Introducing the polar angle $\theta$ and writing

$$
\begin{gathered}
M_{x}=M \sin \theta \cos \omega t ; \quad M_{y}=\mp M \sin \theta \sin \omega t ; \\
M_{z}=M \cos \theta,
\end{gathered}
$$

one can indeed verify immediately that (11) is satisfied if $\theta$ is a constant and chosen such that

$$
\operatorname{tg} \theta=\gamma H_{1} /\left(\gamma H_{0} \mp \omega\right),
$$

with the minus or plus sign before $\omega$, depending upon whether $\gamma$ is positive or negative. If we let

$$
H^{*}=\omega /|\gamma|
$$

denote the "resonance field at frequency $\omega$ " i.e., that field $H$ for which the Larmor frequency $\omega_{L}=\gamma H$ is equal to the frequency $\omega$ of the oscillating field, we can write (16) in the form

$$
\operatorname{tg} \theta=H_{1} /\left(H_{0}-H^{*}\right) \text {. }
$$

Equations (15) represent a solution for which the polarization rotates around the $z$-direction, i.e., around the strong field $H_{0}$ and in such a way that it lies at any instant in the common plane of this field and the effective rotating field (14).

The angle $\theta$ between $H_{0}$ and the polarization follows from (18) to be small, as long as $H_{0}$ is appreciably larger than the resonant field $H^{*}$.

\footnotetext{
${ }^{10}$ F. Bloch and A. Siegert, Phys. Rev. 57, 522 (1940).
} 
The direction of the polarization starts to deviate noticeably from the $z$-direction as the difference $H_{0}-H^{*}$ becomes comparable or small compared to the magnitude $H_{1}$ of the effective rotating field. It is perpendicular to the $z$-direction for $H_{0}=H^{*}$ and for still further decreasing values of $H_{0}$ turns toward the negative $z$-direction, finally pointing in a direction opposite to $H_{0}$ for $H^{*}-H_{0} \ll H_{1}$.

Formulas (15) and (18) can be conveniently rewritten by introducing the difference

$$
\delta=\left(H_{0}-H^{*}\right) / H_{1}=\operatorname{cotg} \theta
$$

between the actual $z$-field $H_{0}$ and its resonance value $H^{*}$ in units of the magnitude $H_{1}$ of the effective rotating field (14) or the half amplitude of the actual oscillating field (5) in the $x$-direction. We have then

$$
\begin{gathered}
M_{x}=M \frac{\cos \omega t}{\left(1+\delta^{2}\right)^{\frac{1}{3}}} ; \quad M_{y}=\mp M \frac{\sin \omega t}{\left(1+\delta^{2}\right)^{\frac{1}{2}}} ; \\
M_{z}=M \frac{\delta}{\left(1+\delta_{z}\right)^{\frac{1}{2}}} .
\end{gathered}
$$

These formulas show clearly the increase of the rotating component of $\mathbf{M}$ upon approach to resonance.

The solution (20) differs essentially from those previously ${ }^{1,2}$. considered, where the nuclear moment can be assumed to be originally in one of the stationary states in which its angle with the $z$-direction is given by $\cos \theta=m / j(m=-j \cdots+j)$ and where it becomes suddenly subjected to the action of an oscillating field, thereby undergoing transitions in which $\theta$ changes through a change of $m$ by \pm 1 . The solution (20) corresponds, in the language of quantum mechanics, not to a single stationary state, but to a "mixture" or linear combination of all stationary states with different values of $m$ and with their amplitudes and phases so adjusted that the expectation values of the components of the angular or magnetic moment are proportional to the values (20). Particularly the $z$-component is not quantized, but has an expectation value which varies continuously with variation of $\delta$. In order to obtain a persistent rotating component of the expectation value of $\mathbf{M}$, as expressed by (20), it is essential, from this point of view, that we are dealing with a "coherent mixture" of states, i.e., that the relative phases of the wave functions, corresponding to the different states, do not undergo any changes. It can be expected, and will be shown later, that any cause which tends to destroy the phase relation, such as the interaction between neighboring nuclei, will diminish the actual observable value of the rotating component.

Our special interest in the particular solution (20) is based upon the fact that, while it has been derived under the assumption that $\omega, H_{0}$, and therefore, $\delta$ are constant, it can be shown to be equally valid, provided that these quantities vary adiabatically, i.e., slowly enough so that

$$
|d \delta / d t| \ll\left|\gamma H_{1}\right| \text {. }
$$

For constant $H_{1}$, i.e., for constant amplitude of the oscillating field, this variation of $\delta$ and, thereby, of the components (20) of the polarization can, according to (19), take place through two different procedures. Either the field $H_{0}$ in the $z$-direction is kept constant and the frequency $\omega$ of the oscillating field is slowly varied, thereby slowly varying the value $H^{*}$ of the resonance field, given by (17); or $\omega$ and therefore $H^{*}$ are kept constant and $H_{0}$ is varied slowly. Both procedures are recommendable in practice, depending upon the circumstances; we shall here assume that the latter procedure is chosen, i.e., that $H_{0}$ varies adiabatically with constant $\omega$.

Whether a variation $d H_{0} / d t$ of $H_{0}$ can be considered as adiabatic or not, depends, according to (19) and (21) upon the half amplitude $H_{1}$ of the $r-f$ field, any given variation being the more adiabatic, the larger $H_{1}$. The condition (21) for adiabatic variation can also be expressed by stating that the $z$-field $H_{0}$ has to pass through an interval, comparable to the "resonance width" $H_{1}$ during a time which is long compared to

$$
t_{1}=1 /\left|\gamma H_{1}\right| \text {. }
$$

As a numerical example, we shall again consider protons with $\gamma=2.66 \times 10^{4}$ c.g.s. ${ }^{11}$ Choosing an amplitude $2 H_{1}$ of 10 gauss, for the r-f field, i.e., with $H_{1}=5$ gauss, we have

$$
t_{1}=7.5 \times 10^{-6} \mathrm{sec},
$$

${ }^{11}$ J. M. B. Kellogg, I. I. Rabi, N. F. Ramsey, Jr., and J. R. Zacharias, Phys. Rev. 56, 728 (1939). 
i.e., the variation is adiabatic, if $H_{0}$ varies by an amount comparable to the "resonance width" of 5 gauss in a time long compared to 7.5 microseconds. It is clear that with the assumed value of $H_{1}$, normal variations of $H_{0}$ can be considered as perfectly adiabatic and that $H_{1}$ has to be chosen very much smaller before this condition is violated.

We shall now make use of the preceding considerations in order to introduce the nuclear induction effect, i.e., the essential features of an experimental arrangement by which a rotating component of the nuclear polarization can be observed through an induced voltage signal.

A sample, containing among others the nuclei under consideration, shall occupy a relatively small volume between the poles of a magnet so that the field $H_{0}$ of the magnet can be considered homogeneous over the extension of the sample, its direction being chosen as the $z$-direction of a right-handed Cartesian coordinate system. An oscillating field with amplitude $2 H_{1}$ and circular frequency $\omega$ shall be produced by an r-f current, passing through a wire which is wound such that the r-f field has an essentially constant amplitude over the sample region and oscillates in the $x$-direction. Immediately surrounding the sample there shall finally be wound a coil with its axis parallel to the $y$-axis, so that an r-f flux in the $y$-direction will manifest itself by an induced $r$-f voltage signal across its terminals. It is such a signal, produced by the rotating component of the nuclear polarization, in which we are primarily interested, and we shall show that one can expect it to be of easily detectable magnitude, with moderate sizes of the sample and under normal laboratory conditions.

In order to estimate the induced $r-f$ voltage we have to consider the expression for $M_{y}$ of Eq. (20). The corresponding value of the induction is

$$
B_{y}=4 \pi M_{y},
$$

and if $N$ turns of the receiver coil surround a cross-sectional area $A$ of the sample, we obtain for the effective flux through the coil

$$
F=4 \pi N A M_{y}=\mp 4 \pi N A M \frac{\sin \omega t}{\left(1+\delta^{2}\right)^{\frac{1}{2}}},
$$

and for the induced voltage $V$ across the ter- minals of the coil

$$
V=-\frac{1}{c} \frac{d F}{d t}= \pm \frac{4 \pi}{c} N A M \omega \frac{\cos \omega t}{\left(1+\delta^{2}\right)^{\frac{1}{2}}}
$$

where the variation of $\delta$ has been considered slow enough so that its time derivative can be neglected compared to that of $\cos \omega t$.

The amplitude of the signal voltage $V$ reaches evidently a maximum at resonance, i.e., for $\delta=0$, so that here the $z$-field $H_{0}$ has, according to (17) and (19), the value $H_{0}=\omega /|\gamma|=H^{*}$. We shall now assume that the sample has been for a sufficiently long time in a field $H_{0}=H>H^{*}$, so that $M$ has reached its thermal equilibrium value, corresponding to that field, and is given by (1) to be $M=\chi H$. If now $H_{0}$ starts to decrease and if our previous assumptions, and particularly that of the constancy of $M$, remain valid, we can substitute this value into (26), thus obtaining

$$
V= \pm \frac{4 \pi}{c} N A \chi H \omega \frac{\cos \omega t}{\left(1+\delta^{2}\right)^{\frac{1}{2}}} .
$$

It has to be observed that it is sufficient for the "equilibrium field" $H$ to be larger than the resonance field $H^{*}$ only by a small percentage, i.e., by several times the relatively weak field $H_{1}$, in order that thermal equilibrium can be established under non-resonant conditions. If we assume this to be actually the case, we can substitute in (27) for $H$ the resonance value $H^{*}=\omega /|\gamma|$. We shall further rewrite the formula (2) for the nuclear susceptibility, using (9) and writing $a=j h / 2 \pi$ for the angular momentum, so that

and finally

$$
\chi=n \frac{j(j+1)}{3 k T}\left(\frac{\gamma h}{2 \pi}\right)^{2},
$$

$$
V=\frac{1}{\pi c} N A n \frac{j(j+1)}{3 k T} h^{2} \gamma \omega^{2} \frac{\cos \omega t}{\left(1+\delta^{2}\right)^{\frac{1}{2}}} .
$$

The plus or minus sign in (27), referring to positive or negative $\gamma$-values, respectively, does not appear any more in this formula, provided that $\gamma$ is here taken algebraically, i.e., positive or negative, depending upon the nuclei under consideration.

We shall now compute from (27) the voltage amplitude $a_{r}=(4 \pi / c) N A \chi H \omega$ at resonance (i.e., 
for $\delta=0$ ) of the signal voltage for protons in water, using the same conditions $(H=10,000 G$, $T=291^{\circ}$ ), which led to the value (4) for $\chi H=M$ $=3.4 \times 10^{-6} G$. The corresponding circular frequency is given by $\omega=|\gamma| H=2.66 \times 10^{4} \times 10,000$ $=2.66 \times 10^{8} \mathrm{sec}^{-1} \cdot(\nu=42$ megacycles. $)$ Assuming the cross-sectional area $A$ of the sample to be $1 \mathrm{~cm}^{2}$ and the number of turns on the receiver coil $N=10$, we obtain the resonance amplitude in volts

$$
\begin{array}{rl}
a_{r}=10^{-8} \times 4 \pi \times 10 \times 3.4 \times 10^{-6} \times 2 & 2.66 \times 10^{-8} \\
= & 1.1 \times 10^{-3} \text { volt. }
\end{array}
$$

We see thus that even under the moderate conditions, here assumed, with linear dimensions of the sample of the order of $1 \mathrm{~cm}$, one can expect from these considerations an $r-f$ signal voltage of the order of a full millivolt, giving a considerable margin in the limits of observation.

Such a margin is desirable, not only because it allows a convenient reduction in the applied values of $H$ and $\omega$, but also because the voltage, given by (27) or (28) represents actually an overestimate. These formulas omit the influence of internuclear and thermal interaction, and we shall see later that they can cause an appreciable reduction from the above estimate.

Not only the magnitude of the induced voltage signal is of interest, but also its sign, i.e., its relative phase with respect to the "driving field" $H_{x}$ of Eq. (5). It is evident that the sign is partly determined by that of $\gamma$, but its determination in Eq. (28) depends also upon the special assumptions under which it was derived, particularly the one expressed in formula (20), that $M_{z}$ has the positive value $M$ for large positive values of $\delta$, i.e., according to (19) for $z$-fields $H_{0}$ appreciably above the resonance field $H^{*}$. This assumption is of course justified, if thermal equilibrium has been established, previous to the passage through resonance, in fields higher than the resonance field. If, on the other hand, the thermal equilibrium was previously established in fields below the resonance field, one must obviously demand that the opposite initial condition is fulfilled, i.e., that $M_{z}$ is positive for negative values of $\delta$. This requires changes in the considerations, leading to Eq. (20), which can immediately be derived from the starting Eq. (11). This equation is linear and homo- geneous in the components of the polarization vector $\mathbf{M}$ so that a change of sign of all components leads necessarily from one solution to another solution. In order to satisfy the condition for positive $M_{z}$ below resonance, it is therefore only necessary to change the sign in the formula (20). The subsequent considerations will all remain valid, except for a change of sign of $M_{y}$ and $V$, leading thus, instead of (28), to the more general formula

$$
V= \pm N A n \frac{j(j+1)}{3 k T} h^{2} \gamma \omega^{2} \frac{\cos \omega t}{\left(1+\delta^{2}\right)^{\frac{1}{2}}},
$$

with the \pm sign referring to the case of the polarization parallel to the $z$-field far above or below resonance, respectively. In using the phase of the induced voltage signal with respect to the driving field for a determination of the sign of $\gamma$, or of the nuclear moment, it is therefore necessary to keep also the relative magnitude of equilibrium field and resonance field in mind, since it likewise affects the phase.

The fact that our formulas express an "indefinite memory" of the nuclear polarization, as to the conditions under which it was created, rests of course upon the initial simplifying assumptions of this section, and particularly upon assumption (1c), that thermal agitation does not affect the nuclei during the time of observation. It is clear from the previous considerations that finite, although possibly rather long, relaxation times will play an important role in the actual behavior of the observable phenomena, and they will be discussed in the following section.

\section{INFLUENCE OF THERMAL AGITATION AND INTERNUCLEAR ACTION}

The considerations of the preceding section can be regarded only as qualitative, since they are based upon the assumption (1) that all changes of nuclear orientation are due to the external fields. It implies the omission of three major internal actions, mentioned under (1a), (1b), and (1c), of Section 3, which are likewise responsible for changes of orientation.

The first action is that of atomic moments upon the nuclei. Its importance depends evidently upon the substance under consideration, 
i.e., upon whether such moments are actually present or not. There are indeed many substances (e.g., water) where it is safe to assume their absence, i.e., where the electronic spin moments will be paired off and where orbital moments which may be present in the free atoms or molecules are quenched because of intermolecular action. While assumption (1a) is justified under these circumstances, one has to introduce major changes in the presence of permanent atomic moments. The fields due to these moments and acting upon the nuclei will generally be considerably stronger than the external fields, and we do not have to deal in first approximation with independent nuclei, but with nuclear moments which are strongly coupled to the atomic frame. The situation is analogous to that investigated by Rabi and his collaborators $^{12}$ for free molecules, where the $r-f$ field causes transitions between hyperfine structure levels. Except for a broadening of these levels, due to interatomic forces, similar phenomena can be expected in liquids and solids (e.g., in the salts of the rare earths), but we shall not enter here upon their discussion and shall restrict ourselves to the case where permanent atomic moments are absent.

There remain then the internal fields due to thermal agitation and internuclear action which have to be considered. Although both these fields are usually considerably weaker than the external applied fields, they are of importance because of their cumulative effects over longer periods of time. We shall not attempt here to give a rigorous quantum-mechanical theory of these effects; an excellent start for such a theory has been made in the papers mentioned in reference 8. Instead, we shall restrict ourselves to a semi-macroscopic description, trying to introduce into Eq. (11) for the macroscopic polarization such modifications as are necessary to account for the principal features of these effects.

To arrive at these modifications we shall consider a finite polarization $\mathbf{M}$ to exist at a certain moment and shall separately investigate the changes which it will undergo due to thermal agitation and internuclear action. Although there is a certain similarity insofar as both represent random actions upon the individual nuclei, there is this essential difference, that only thermal perturbations can change the energy of the total spin system, while internuclear interactions leave this energy unchanged.

The dominant part of the total spin energy $E$ is caused to the strong field $H_{0}$ in the $z$-direction and can be written in the form

$$
E=-H_{0} M_{z} \text {. }
$$

Major changes of the total energy are therefore necessarily due to a change of the $z$-component of the polarization and it will be the thermal perturbations which will be responsible for these changes. The equilibrium value which $M_{z}$ will approach under the influence of thermal perturbations is given by

$$
M_{0}=\chi H_{0} .
$$

If at any time $M_{z} \neq M_{0}$, it will approach this value exponentially with a characteristic time constant $T_{1}$, which we shall call the "thermal" or "longitudinal" relaxation time, and we can describe the rate of change of $M_{z}$, due to thermal perturbations alone, by the differential equation

$$
\dot{M}_{z}=-\left(M_{z}-M_{0}\right) / T_{1}
$$

with the stationary solution $M_{z}=M_{0}$.

The actual value of $T_{1}$ is very difficult to predict for a given substance; it depends delicately, not only upon the thermal motion of the atoms which is quite different in gases, liquids, and solids, but also upon their electronic structure and its modification, due to interatomic forces. Rough estimates can easily lead to relaxation times of many seconds or even hours. We shall see below that such long relaxation times can be inconvenient for the observation of the induction effect. It is recommendable, in this case, to add to the substance a certain percentage of paramagnetic atoms or molecules. They will essentially act as catalysts, with the relatively strong fields of their permanent moments greatly shortening the relaxation time $T_{1}$, even if they are present in a small percentage and do not otherwise affect the nuclei under consideration.

The fields which are due to neighboring nuclei, also contribute to the establishment of the equilibrium because of their thermal agitation. These fields are so small that they alone would normally lead to extraordinarily long thermal relaxation 
times with their influence upon the actual value $T_{1}$ being negligible. Internuclear actions can, however, be of importance for the changes of the other two components $M_{x}$ and $M_{y}$ of the polarization in which we are equally interested. The fact that the nuclei with their moments participate in the thermal agitation is indeed of minor importance and represents only a small correction in their effect upon these components, since changes of $M_{x}$ and $M_{y}$ do not affect the energy (30) of the total spin system in the field. These changes can, therefore, take place without the necessity of transferring part of the spin energy $E$ into kinetic energy of the atoms and it is permissible, in this respect, to consider the nuclei at rest and to neglect their comparatively slow motion.

Processes in which the total energy of the spin system does not change and which therefore affect only the components of the polarization which are transversal to the field are not necessarily due to internuclear forces alone. Small and irregular inhomogeneities of the $z$-field $H_{0}$ and the presence of other moments, such as those of paramagnetic ions in solution, will cause similar effects. It will in fact be permissible, for a qualitative discussion, to describe all these effects, including internuclear actions, by an "effective irregularity" of the $z$-field of order $H^{\prime}$ and through this field by a "transversal relaxation time"

$$
T_{2}=1 /|\gamma| H^{\prime},
$$

which it takes for $M_{x}$ and $M_{y}$ to be appreciably affected.

The magnitude of $T_{2}$ can be easily estimated if $H^{\prime}$ is due to neighboring nuclei whose motion can be neglected. With $\mu$ being the magnetic moment of a neighboring nucleus and $r$ being its distance one will expect

$$
H^{\prime} \cong \mu / r^{3}
$$

Choosing $\mu=10^{-23}$ c.g.s. and $r=2 \times 10^{-8} \mathrm{~cm}$ this leads to

$$
H^{\prime}=1 \text { gauss }
$$

and with $\gamma=10^{4} \mathrm{c} . g . s$. through (33) to

$$
T_{2}=10^{-4} \mathrm{sec} \text {. }
$$

To give a reliable estimate of $T_{2}$ in more general cases requires a more detailed investigation of the mechanisms involved and will not be attempted here. The special case leading to (36) will merely be considered as an illustration for the fact that the transversal relaxation time $T_{2}$ can be many orders of magnitude smaller than the longitudinal time $T_{1}$ and that serious errors may be committed by assuming $T_{1}=T_{2}$. There are, on the other hand, also cases where this equality is justified, particularly those where both relaxation times are due to impacts which last during a time short compared to the Larmor period, so that the distinction between collisions which change the spin energy and those which leave it unaltered becomes immaterial.

In order to obtain a qualitative description of the total change of the nuclear polarization $\mathbf{M}$ with time we shall now introduce such terms for its rate of change which contain the essential features of a longitudinal and transversal relaxation time and which, at the same time, are chosen so as to complicate the analysis as little as possible. For this purpose we shall assume that in analogy to the change (32) of the longitudinal component, the change of $M_{x}$ and $M_{y}$ will likewise be of an exponential character, governed by the equations

$$
\begin{aligned}
& M_{x}=-\left(1 / T_{2}\right) M_{x}, \\
& M_{y}=-\left(1 / T_{2}\right) M_{y}
\end{aligned}
$$

and that the total rate of change of $\mathbf{M}$ is obtained by adding to the expression (11), which takes into account the action of external fields only, the changes (32) and (37) of its components due to internal actions. The appearance of the same time constant $T_{2}$ in both Eqs. (37) is justified if one considers the substance to be isotropic. We obtain then the following differential equations for the three components of $\mathbf{M}$ :

$$
\begin{aligned}
& \dot{M}_{x}-\gamma\left(M_{y} H_{z}-M_{z} H_{y}\right)+\frac{1}{T_{2}} M_{x}=0, \\
& \dot{M}_{y}-\gamma\left(M_{z} H_{x}-M_{x} H_{z}\right)+\frac{1}{T_{2}} M_{y}=0, \\
& \dot{M}_{z}-\gamma\left(M_{x} H_{y}-M_{y} H_{x}\right)+\frac{1}{T_{1}} M_{z}=\frac{1}{T_{1}} M_{0} .
\end{aligned}
$$

The components of the external field are given by Eq. (5) and we shall replace, as in Section 3, the actual oscillating $x$-component by the ef- 
fective rotating component (14), so that we have

$H_{x}=H_{1} \cos \omega t ; \quad H_{y}=\mp H_{1} \sin \omega t ; \quad H_{z}=H_{0}$

with the two signs of $H_{y}$ referring to positive and negative values of $\gamma$, respectively.

It is convenient to introduce instead of $M_{x}$ and $M_{y}$ two new variables $u$ and $v$ through

$$
\begin{aligned}
& M_{x}=u \cos \omega t-v \sin \omega t, \\
& M_{y}=\mp(u \sin \omega t+v \cos \omega t) .
\end{aligned}
$$

We shall further choose the time scale in units of $1 /|\gamma| H_{1}$ by introducing the dimensionless quantities:

$$
\begin{gathered}
\tau=|\gamma| H_{1} t ; \quad \alpha=\frac{1}{|\gamma| H_{1} T_{1}} ; \quad \beta=\frac{1}{|\gamma| H_{1} T_{2}} ; \\
\delta=\frac{|\gamma| H_{0}-\omega}{|\gamma| H_{1}}
\end{gathered}
$$

where $|\gamma|$ is the absolute value of $\gamma$. Using (39)-(41), one can then write the Eqs. (38) in the form :

$$
\begin{aligned}
d u / d \tau+\beta u+\delta v & =0, \\
d v / d \tau+\beta v-\delta u+M_{z} & =0, \\
d M_{z} / d \tau+\alpha M_{z}-v & =\alpha M_{0} .
\end{aligned}
$$

The Eqs. (38) differ from the three component Eqs. (11) of Section 3, insofar as they contain on their left sides the "damping terms" inversely proportional to $T_{1}$ and $T_{2}$ and that on the right side of (38c) there appears an inhomogeneous term, proportional to the equilibrium polarization $M_{0}=\chi H_{0}$. Their solution offers no difficulty, especially if $\delta$ is a slowly varying quantity so that they appear in the form (42) as a system of linear differential equations with almost constant coefficients; a more detailed discussion will be reserved for a later occasion. We shall here give directly a particular solution which is of special interest for our present purposes; its validity can be verified, provided that the variation of $\delta$ is adiabatic in the sense of Section 3 and that both quantities $\alpha$ and $\beta$ of (41) are assumed to be small compared to unity. The first condition implies $|d \delta / d \tau| \ll 1$; it is identical with Eq. (21) and will be the more closely satisfied for any given variation with time of $\delta$, the larger the amplitude of the oscillating field. In order to have $\alpha \ll 1$, $\beta \ll 1$, it is necessary, according to (41), that either the relaxation times $T_{1}$ and $T_{2}$ are sufficiently large or that the amplitude $2 H_{1}$ of the oscillating field is sufficiently large.

One can normally expect the transverse relaxation time $T_{2}$ to be smaller than the longitudinal $T_{1}$ or $\alpha<\beta$. The assumption of "large amplitudes" implies then, according to (41), that

$$
H_{1} \gg \frac{1}{|\gamma| T_{2}}
$$

or, according to (33),

$$
H_{1} \gg H^{\prime} .
$$

Using for a numerical example the value (35) of 1 gauss for $H^{\prime}$, we see therefore that an amplitude of $2 H_{1}=10$ gauss can already be considered as "large" although it will normally be still very small compared to the strong field $H_{0}$ (of the order of several thousand gauss) in the $z$-direction. It is in this case $\beta=0.2$ and no serious errors are committed in neglecting correction terms in $\alpha$ and $\beta$.

With the three quantities $|d \delta / d \tau|, \alpha$ and $\beta$ small compared to unity our particular solution of (38) can be written in the convenient form

$$
\begin{aligned}
& M_{x}=\frac{M}{\left(1+\delta^{2}\right)^{\frac{1}{2}}} \cos \omega t, \\
& M_{y}=\mp \frac{M}{\left(1+\delta^{2}\right)^{\frac{1}{2}}} \sin \omega t, \\
& M_{z}=\frac{M \delta}{\left(1+\delta^{2}\right)^{\frac{1}{2}}},
\end{aligned}
$$

with

$$
\begin{gathered}
\delta=\delta(t)=\frac{H_{0}(t)-H^{*}}{H_{1}} ; \quad H^{*}=\frac{\omega}{|\gamma|}, \\
M=M(t)=\frac{1}{T_{1}} \\
\times \int_{-\infty}^{t} \frac{\delta\left(t^{\prime}\right) \exp \left\{-\left[\theta(t)-\theta\left(t^{\prime}\right)\right]\right\} M_{0}\left(t^{\prime}\right)}{\left[1+\delta^{2}\left(t^{\prime}\right)\right]^{\frac{1}{2}}} d t^{\prime}, \\
M_{0}\left(t^{\prime}\right)=\chi H_{0}\left(t^{\prime}\right), \\
\theta(t)-\theta\left(t^{\prime}\right)=\frac{1}{T_{1}} \int_{t^{\prime}}^{t} \frac{\delta^{2}\left(t^{\prime \prime}\right)+T_{1} / T_{2}}{1+\delta^{2}\left(t^{\prime \prime}\right)} d t^{\prime \prime}
\end{gathered}
$$




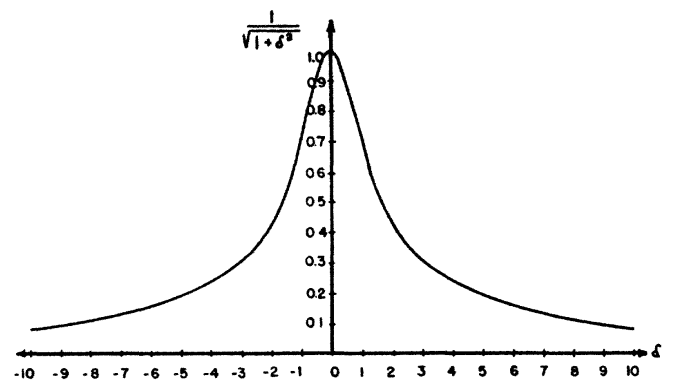

FIg. 1. Schematic representation of the voltage amplitude in the case of rapid passage. The abscissa $\delta$ is the deviation of the actual field from the resonance field in units of the half-amplitude of the oscillating field. The ordinate is proportional to the amplitude of the induced voltage and the $y$-component of the nuclear polarization.

The general solution of (38) is of course obtained by adding to (44) arbitrary multiples of the homogeneous solutions, their factors being determined by the initial conditions. These solutions can be seen to decrease exponentially with time so that it is permissible to omit them after a sufficient time has elapsed. In practice it is only the solution (44) which has to be considered, since it corresponds to the situation where the equilibrium polarization $M_{0}$ was zero before a sufficient time in the past, i.e., where the actual conditions have been obtained by adiabatic change, starting from an originally unpolarized sample.

The Eqs. (44) have the same form as the Eqs. (20) of Section 3, with the difference that $M$ is not an arbitrary constant but is generally a function of time, given by (46). It has to be observed that while its absolute value $|M|$ still represents the instantaneous magnitude of the polarization, the quantity $M$ itself is not necessarily positive but can have both signs, depending upon the positive or negative values which $\delta$ in the integrand of (46) has assumed in the past. We are primarily interested in the behavior of the polarization near resonance and it is interesting to observe that $M$ may here be considered as essentially constant or appreciably variable, depending upon the speed of variation of $\delta$, i.e., of either $H_{0}$ or $\omega$ in comparison to the relaxation times.

Formula (46) shows in fact that the value of $M$ at any time is determined by the past history and that the more remote a past is of importance, the slower the decrease of the exponential. The functional dependence of $M$ upon time and particularly its behavior near resonance will be primarily determined by the relative change of $\delta$ and of the exponential in the integrand of (46).

We shall speak of the limiting case of "rapid passage" if $\delta$ varies near resonance by an amount of order unity during a time short compared to the variation of the exponential. This case is the more approximately realized the more rapid the change of $\delta$ or, according to (48), the longer the relaxation times $T_{1}$ and $T_{2}$. It can be easily seen with the use of Eqs. (41) that because of the assumed smallness of $\alpha$ and $\beta$ such a relatively rapid change of $\delta$ is compatible with the adiabatic condition $|d \delta / d t| \ll 1$. In the neighborhood of resonance $M$ can be considered in this case to be essentially constant, its actual value depending mostly upon the values which $M_{0}$ and $\delta$ have assumed an appreciable time before approach of resonance conditions.

The amplitude of $M_{y}$ is then proportional to $1 /\left(1+\delta^{2}\right)^{\frac{1}{2}}$ and is schematically represented in Fig. 1. The simplified situation, considered in Section 3, is in fact a special case of "rapid passage" or long relaxation time. It is assumed here that $H_{0}$ has been held fixed for a long time at a value $H$, far from resonance, so that $|\delta| \gg 1$ with a subsequent establishment of resonance conditions during a time, very short compared to $T_{1}$. The main contribution to (46) arises then from past times $t^{\prime}$, where $M_{0}$ had the constant value $\chi H$ and $\delta^{2}$ had a value large compared to unity. If we assume, besides, for this value $\delta^{2} \gg T_{1} / T_{2}$ or if we assume, irrespective of $\delta$ that $T_{1}=T_{2}$ we obtain from (48)

$$
\theta(t)-\theta\left(t^{\prime}\right)=\frac{t-t^{\prime}}{T_{1}}
$$

and from (46)

$M= \pm \frac{\chi H}{T_{1}} \int_{-\infty}^{t} \exp \left\{-t-t^{\prime} / T_{1}\right\} d t^{\prime}= \pm \chi H$.

The magnitude of $M$ corresponds thus, as was to be expected, to the field $H$, to which the sample was sufficiently long subjected. The plus or minus sign in (49) has to be taken, depending on whether this field was stronger or weaker than the resonance field, i.e., whether during action of this field, far from resonance, we had $\delta /\left(1+\delta^{2}\right)^{1}=+1$ 
or $\delta /\left(1+\delta^{2}\right)^{\frac{1}{2}}=-1$. This duplicity of the resulting sign of $M_{y}$ upon approach of resonance from "above" or "below" was discussed in Section 3 and must not be confused with the other duplicity, expressed in (44b) and depending upon the sign of $\gamma$.

The opposite limiting case, to be considered, is that of "slow passage" through resonance or short relaxation times, where the main variation in the integrand of (46) is caused by the exponential. It can be treated by writing

$$
\frac{\partial}{\partial t^{\prime}} \exp -\left[\theta(t)-\theta\left(t^{\prime}\right)\right]=\frac{\partial \theta\left(t^{\prime}\right)}{\partial t^{\prime}} \exp -\left[\theta(t)-\theta\left(t^{\prime}\right)\right]
$$

or using (48)

$$
\begin{aligned}
\exp -\left[\theta(t)-\theta\left(t^{\prime}\right)\right] \\
=T_{1} \frac{1+\delta^{2}\left(t^{\prime}\right)}{\delta^{2}\left(t^{\prime}\right)+\left(T_{1} / T_{2}\right)} \frac{\partial}{\partial t^{\prime}} \exp -\left[\theta(t)-\theta\left(t^{\prime}\right)\right]
\end{aligned}
$$

so that by partial integration, we obtain from (48)

$$
\begin{aligned}
M(t)= & \frac{1+\delta^{2}(t)}{\delta^{2}(t)+\left(T_{1} / T_{2}\right)} \frac{\delta(t) M_{0}(t)}{\left[1+\delta^{2}(t)\right]^{\frac{1}{2}}} \\
& \quad-\int_{-\infty}^{t} \exp -\left[\theta(t)-\theta\left(t^{\prime}\right)\right] \frac{d}{d t^{\prime}} \\
& \times\left[\frac{1+\delta^{2}\left(t^{\prime}\right)}{\delta^{2}\left(t^{\prime}\right)+\left(T_{1} / T_{2}\right)} \frac{\delta\left(t^{\prime}\right) M_{0}\left(t^{\prime}\right)}{\left[1+\delta^{2}\left(t^{\prime}\right)\right]^{\frac{1}{2}}}\right] d t^{\prime} .
\end{aligned}
$$

The last integral becomes evidently negligible if the variation of $\delta$ and $M_{0}$ with time is sufficiently slow. Keeping only the dominant first term in (50) and substituting into (44), we find thus:

$$
\begin{aligned}
& M_{x}(t)=\frac{M_{0}(t) \delta(t)}{\delta^{2}(t)+T_{1} / T_{2}} \cos \omega t, \\
& M_{y}(t)=\mp \frac{M_{0}(t) \delta(t)}{\delta^{2}(t)+T_{1} / T_{2}} \sin \omega t, \\
& M_{z}(t)=\frac{M_{0}(t) \delta^{2}(t)}{\delta^{2}(t)+T_{1} / T_{2}} .
\end{aligned}
$$

In contrast to the case of rapid passage, where $M$ can be considered essentially constant and where the amplitudes of $M_{x}$ and $M_{y}$ reach, according to (44) their maximum at resonance, i.e., for $\delta=0$, it is seen from (51) that in the case of slow passage all three components of the polarization vanish at resonance. Since $M_{0}$ varies only little in the neighborhood of resonance, it is permissible to replace it by the equilibrium polarization $M^{*}$ at the resonance field $H^{*}$, i.e., to write

$$
M_{0}=M^{*}=\chi H^{*}=\chi \frac{\omega}{|\gamma|} .
$$

The amplitudes of $M_{x}$ and $M_{y}$ are then given by

$$
a_{x, y}=\chi \frac{\omega}{|\gamma|} f(\delta)
$$

where

$$
f(\delta)=\frac{\delta}{\delta^{2}+T_{1} / T_{2}}
$$

We have plotted this function in Fig. 2. It assumes its extremum values

$$
\text { for } \quad \begin{aligned}
f(\delta) & = \pm \frac{1}{2}\left(T_{2} / T_{1}\right)^{\frac{1}{2}} \\
\delta & = \pm\left(T_{1} / T_{2}\right)^{\frac{1}{2}}
\end{aligned}
$$

and shows qualitatively different behavior from the simple maximum, to be expected in the case of rapid passage and represented in Fig. 1.

The case of "slow passage" can also be treated directly without the restriction $\alpha \ll 1, \beta \ll 1$ which led to the solution (44) and (51). With $\delta$ and $M_{0}$ so slowly varying that they can be considered as practically constant one can in this case obtain a solution of the Eqs. (42) by assuming $u, v$, and $M_{z}$ likewise as practically constant, i.e., by neglecting their derivatives. With $d u / d \tau=d v / d \tau$

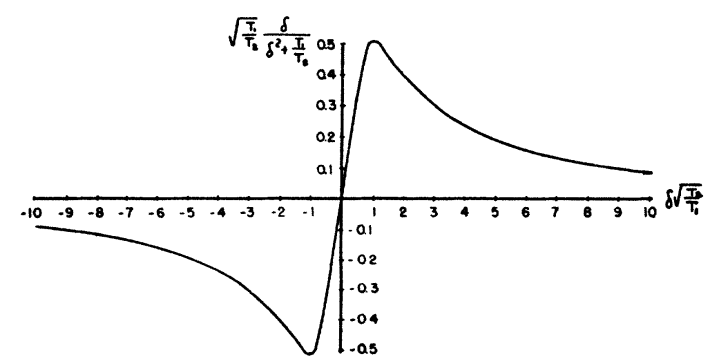

FIG. 2. Schematic representation of the voltage amplitude in the case of slow passage. $T_{1}$ and $T_{2}$ are the longitudinal and transversal relaxation times, respectively, and the scale is chosen such as to make the plot independent of their values. The significance of abscissa and ordinate is otherwise the same as in Fig. 1. 
$=d M_{z} / d \tau=0$ one obtains directly from the Eqs. (42)

$$
\begin{gathered}
u=\frac{\delta}{\beta^{2}+\delta^{2}+\beta / \alpha} M_{0}, \\
v=-\frac{\beta}{\beta^{2}+\delta^{2}+\beta / \alpha} M_{0}, \\
M_{z}=\frac{\beta^{2}+\delta^{2}}{\beta^{2}+\delta^{2}+\beta / \alpha} M_{0} .
\end{gathered}
$$

Using $\beta / \alpha=T_{1} / T_{2}$ and neglecting for small values of $\beta, v$ and the term $\beta^{2}$ in the numerators this is through (40) identical with the solution (51).

It is interesting, however, to investigate more closely this solution for arbitrary $\beta$, particularly since it allows in the case of slow passage a direct comparison between the condition, favorable for nuclear induction on one side and resonance absorption on the other. Using the Eqs. (41) and writing $|\gamma| H_{0}-\omega=\Delta \omega$ for the deviation of the resonance frequency from $\omega$ we can also write

$$
\begin{gathered}
u=\frac{|\gamma| H_{1} T_{2}^{2} \Delta \omega}{1+\left(T_{2} \Delta \omega\right)^{2}+\left(\gamma H_{1}\right)^{2} T_{1} T_{2}} M_{0}, \\
v=-\frac{|\gamma| H_{1} T_{2}}{1+\left(T_{2} \Delta \omega\right)^{2}+\left(\gamma H_{1}\right)^{2} T_{1} T_{2}} M_{0}, \\
M_{z}=\frac{1+\left(T_{2} \Delta \omega\right)^{2}}{1+\left(T_{2} \Delta \omega\right)^{2}+\left(\gamma H_{1}\right)^{2} T_{1} T_{2}} M_{0} .
\end{gathered}
$$

For nuclear induction it is evidently recommendable to have $u$ as large as possible. Its maximum is obtained for

$$
\Delta \omega=\frac{1}{T_{2}}\left[1+\left(\gamma H_{1}\right)^{2} T_{1} T_{2}\right]^{\frac{1}{2}}
$$

and has here the value

$$
u_{\max }=\frac{|\gamma| H_{1} T_{2}}{\left[1+\left(\gamma H_{1}\right)^{2} T_{1} T_{2}\right]^{\frac{1}{2}}} M_{0} .
$$

This value again increases monotonically with $H_{1}$ and for

becomes

$$
H_{1} \gg \frac{1}{|\gamma|\left(T_{1} T_{2}\right)^{\frac{1}{2}}}
$$

$$
u_{\max \max }=\left(T_{2} / T_{1}\right)^{\frac{1}{2}} M_{0} .
$$

The condition that the r-f field amplitude $H_{1}$ is large enough to obtain the highest possible $u$ is according to (41) equivalent with $\alpha \beta \ll 1$ and also actually assumed in the derivation of (51) since it is valid only for $\alpha \ll 1, \beta \ll 1$.

To obtain maximum absorption, it is on the other hand necessary to make $v$ as large as possible, since it is this quantity which, through (40a) determines the out of phase part of $M_{x}$. v has its maximum

$$
v_{\max }=-\frac{|\gamma| H_{1} T_{2}}{1+\left(\gamma H_{1}\right)^{2} T_{1} T_{2}} M_{0}
$$

for $\Delta \omega=0$. Unlike $u_{\max }$ this quantity does not increase monotonically for increasing $H_{1}$, but decreases for large values of $H_{1}$. As observed by Purcell, Torrey, and Pound ${ }^{6}$ it is therefore not advisable for absorption experiments to use too intensive $r$-f fields. The best possible choice is

$$
H_{1}=\frac{1}{|\gamma|\left(T_{1} T_{2}\right)^{\frac{1}{2}}}
$$

and yields

$$
v_{\max \max }=\left(T_{2} / T_{1}\right)^{\frac{1}{2}} M_{0} .
$$

It seems satisfactory that the maximum values (60) and (63) of the two decisive quantities $u$ and $v$ is thus the same, although to obtain the one, necessary for induction, requires the $r-f$ field amplitude to be large compared to the "critical amplitude" $1 /|\gamma|\left(T_{1} T_{2}\right)^{\frac{1}{2}}$ whereas the other, necessary for absorption requires it to be equal to this quantity.

It is evident from (44b) and (46) that the magnitude of the signal, induced by the component $M_{y}$ of the nuclear polarization, depends not only upon the susceptibility $\chi$ but also, in a rather complicated way, upon the relaxation times and upon the magnitude and speed of variation of $\delta$. The expression (28) for the induced voltage which was derived in Section 3 under simplifying assumptions will usually not correspond to the observed values but represents merely an estimate to be approached under favorable conditions. The special case of "rapid" passage, considered before and leading to the expression (49) of $M$ represents such a favorable condition, and it is only in this limiting case that 
(28) represents the actually observable value of the induced voltage.

The maximum value of $M$ and thereby of the induced voltage signal at resonance must be expected normally to be smaller than (49) and in fact becomes the smaller for given $T_{2}$, and under otherwise equal conditions, the longer the relaxation time $T_{1}$. In the limit of very large $T_{1}$ one obtains from (48)

$$
\theta(t)-\theta\left(t^{\prime}\right)=\frac{1}{T_{2}} \int_{t^{\prime}}^{t} \frac{d t^{\prime \prime}}{1+\delta^{2}\left(t^{\prime \prime}\right)},
$$

which is independent of $T_{1}$, so that, according to (46) $M$ becomes inversely proportional to $T_{1}$. One may say, in this sense, that too long relaxation times $T_{1}$ are unfavorable for the observation of the effect unless the variation of $\delta$ with time is changed so as to re-establish favorable conditions. It is otherwise recommendable to use samples in which $T_{1}$ is as short as possible, possibly by adding paramagnetic catalysts as previously mentioned. As $T_{1}$ becomes sufficiently short, one will approach the case of "slow passage" mentioned above and represented in Fig. 2. While it will not be possible to have actually $T_{1} \ll T_{2}$ it $\mathrm{s}$ seen from (54) that the maximum signal will still increase with decreasing $T_{1}$, the optimum being reached for $T_{1}=T_{2}$.

\section{CONCLUSIONS}

While the methods of molecular beams and of nuclear induction have a common ground of investigation it is evident that neither one makes the other superfluous. There remains a large complex of interesting nuclear experiments to be done, for which molecular beams are essential, particularly those which call for matter of extremely low density and the least possible interference of neighboring atoms or molecules. There are, on the other hand, many problems which become accessible or which can be more conveniently solved through nuclear induction and some of these will be mentioned here:

(1) The exact comparison of the magnetic moments of the neutron, the proton, and the deuteron is at present one of the most interesting problems, concerning nuclear forces. The main difficulty in this comparison was until now the sufficiently accurate calibration of the resonance field. It can be completely avoided by repeating the experiment of Alvarez and Bloch ${ }^{2}$ for neutrons and by observing through nuclear induction s.multaneously and in the same field the resonances of protons and deuterons. The problem of comparison of their magnetic moments is thus reduced to that of their respective resonance frequencies and can be solved with high accuracy. It was indeed with this experiment in mind, and while searching for a suitable method of comparison, that the author was led to the thought of nuclear induction, and preparations are now under way at Stanford to carry out the measurement in the near future.

(2) One of the difficulties in the determination of the gyromagnetic ratios of many nuclei by molecular beams is that of finding suitable detectors. The method of nuclear induction is free from this obstacle and should be soon applied to all elements for which this determination is of interest.

(3) While even in its very initial stage, nuclear induction was observed with a sample of 100 milligrams, there are good reasons to believe that the sensitivity can still be greatly increased. This offers the possibility to observe the effect not only in liquids and solids but also in gases under no excessive pressure. With only small amounts of matter necessary for its performance, the experiment offers a convenient way of isotope analysis and particularly also for its application to radioactive nuclei.

(4) It was shown in Section 4 that the induced signals to be expected depend not only upon the nuclear susceptibility but also upon the relaxation times. By suitable choice of the variation with time of resonant field or frequency, it is thus possible to measure these quantities separately. The study of nuclear relaxation times is of interest not only as an experimental method to investigate the establishment of thermal equilibrium, but also because of its importance for reaching extremely low temperatures through the nuclear magnetocaloric effect. While even the information gained at room temperature is valuable, it is clear that it can be greatly enlarged by studying the temperature dependence of the effect and particularly its behavior at low temperatures. It is in this same respect that the effect of paramagnetic catalysts, mentioned in Sections 1 and 4, seems of considerable interest. 
(5) As in comparing the moments of neutron, proton, and deuteron, nuclear induction can well be developed as a simple and practical method to calibrate and measure high magnetic fields with great accuracy, and to apply it, for example, in the construction of cyclotrons and mass spectrographs.
There are unquestionably more problems which will become tangible in further development of the new electromagnetic effects. The fact that they are simple to obtain and require only very modest equipment should make it possible for many investigators to enter this field of research.

\title{
The Nuclear Induction Experiment
}

\author{
F. Bloch, W. W. Hansen, and M. Packard \\ Stanford University, California
}

(Received July 19, 1946)

\begin{abstract}
The phenomenon of nuclear induction has been studied experimentally. The apparatus used is described, both as to principle and detail. Experiments have been carried out in which the signals from protons contained in a variety of substances were observed. The results show the role played by the relaxation time, which was found to vary between about $10^{-5}$ second and many seconds.
\end{abstract}

\section{INTRODUCTION}

$I^{1}$ $\mathrm{N}$ this paper we aim to describe certain experiments designed to study the phenomenon of nuclear induction, the theory of which is described in the preceding paper. While it will be assumed that this paper, hereafter referred to as I, is familiar to the reader, we will not hesitate to repeat those few formulae which are of immediate importance for the present paper. We start, then, by giving the reasons for the selection of the general methods used and follow this by a brief description of specific apparatus. Finally, the results are described in detail.

\section{METHOD}

If matter be placed in a magnetic field $H_{0}$, in the $z$ direction, the nuclear magnetic moments will tend to orient themselves parallel to the field. In matter of normal density, thermal equilibrium may be established, in which case there will be a paramagnetic polarization in the $z$ direction

$$
M_{0}=\chi H_{0} .
$$

If now we superimpose an oscillating magnetic field in the $x$ direction

$$
H_{x}=2 H_{1} \cos \omega t,
$$

we can expect the polarization vector to deviate appreciably from the $z$ direction if the $z$ field $H_{0}$ approaches the resonance value $H^{*}$ given by

$$
|\gamma| H^{*}=\omega
$$

where $\gamma$, the gyromagnetic ratio, is the ratio of nuclear moment to angular momentum characteristic of the nuclei under consideration. Quantitatively, the result of this deviation may be described by a macroscopic polarization vector $\mathbf{M}$ with components given by Eq. (44) of I,

$$
\begin{aligned}
& M_{x}=\frac{M}{\left(1+\delta^{2}\right)^{\frac{1}{2}}} \cos \omega t, \\
& M_{y}=\mp \frac{M}{\left(1+\delta^{2}\right)^{\frac{1}{3}}} \sin \omega t, \\
& M_{z}=\frac{M}{\left(1+\delta^{2}\right)^{\frac{1}{2}}},
\end{aligned}
$$

where

$$
\delta=\left(H_{0}-H^{*}\right) / H_{1}
$$

is the deviation of $z$ field $H_{0}$ from its resonance value $H^{*}$ in units of the half-amplitude $H_{1}$ of the oscillating $x$ field. The ambiguous sign in (4b) is to allow for the possibility of either positive or negative values of $\gamma$. The quantity $M$ depends in 